\title{
Markov approximation of arbitrary random field on homogeneous trees
}

\author{
Weicai Peng ${ }^{1 *}$, Weiguo Yang $^{2}$ and Peishu Chen ${ }^{1}$
}

\author{
* Correspondence: \\ weicaipeng@126.com \\ ${ }^{1}$ Department of Mathematics, \\ Chaohu University Chaohu, 238000, \\ P.R. China \\ Full list of author information is \\ available at the end of the article
}

\begin{abstract}
In this article, we establish a class of small deviation theorems for functionals of random fields and the strong law of large numbers for the ordered couple of states for arbitrary random fields on homogenous trees. A known result is generalized in this article.

2010 Mathematics Subject Classification: 60J10; 60F15.

Keywords: Small deviation theorems, homogeneous tree; random field, strong law of large numbers
\end{abstract}

\section{Introduction}

By a tree, $T$ we mean an infinite, locally finite, connected graph with a distinguished vertex $o$ called the root and without loops or cycles. We only consider trees without leaves. That is, the degree of each vertex (except $o$ ) is required to be at least two. Let $\sigma$, $\tau$ be vertices of a tree. Write $\tau \leq \sigma$ if $\tau$ is on the unique path connecting $o$ to $\sigma$, and $|\sigma|$ for the number of edges on this path. For any two vertices, $\sigma, \tau$, denote by $\sigma \wedge \tau$ the vertex farthest from $o$ satisfying $\sigma \wedge \tau \leq \sigma, \sigma \wedge \tau \leq \tau$.

Some tree models have been introduced in the literature. We say a homogeneous tree, namely, a Bethe tree, on which each vertex has $N+1$ neighboring vertices. Another homogeneous tree is a rooted Cayley tree $T_{C, N^{-}}$the root has $N$ neighbors, and the other vertices have $N+1$ neighbors.

The set of all vertices with distance $n$ from the root $o$ is called the $n$th generation (or $n$th level) of $T$, which is denoted by $L_{n}, L_{0}=\{0\}$. We denote by $T_{(m)}^{(n)}$ the subtree of a tree $T$ containing the vertices from level $m$ to level $n$, especially $T^{(n)}$ the subtree of a tree $T$ containing the vertices from level 0 to level $n$. Let $t$ be a vertex of $T$, predecessor of the vertex $t$ is another vertex which is the nearest from $t$ on the unique path from root $o$ to $t$. We denote the predecessor of $t$ by $1_{t}$, the predecessor of $1_{t}$ by $2_{t}$, the predecessor of $n_{t}$ by $(n+1)_{t}$, and $0_{t}=t$, where $n=0,1,2, \ldots$. We also say that $n_{t}$ is the $n$th predecessor of $t . X^{A}=\left\{X_{t}, t \in A\right\}$ is a stochastic process indexed by a set $A$, and denoted by $|A|$ the number of vertices of $A ; x^{A}$ is the realization of $X^{A}$.

Let $(\Omega, \mathcal{F})$ be a measurable space, and $\left\{X_{t}, t \in T\right\}$ be a collection of random variables defined on $(\Omega, \mathcal{F})$ and take value in states space $S$.

Definition 1 (Tree-indexed Markov chains) Let $T$ be a tree, $S$ be a finite space, and $\left\{X_{\sigma}, \sigma \in T\right\}$ be a collection of $S$-valued random variables defined on the probability space $(\Omega, \mathcal{F}, P)$. Let

(C) 2012 Peng et al; licensee Springer. This is an Open Access article distributed under the terms of the Creative Commons Attribution License (http://creativecommons.org/licenses/by/2.0), which permits unrestricted use, distribution, and reproduction in any medium, provided the original work is properly cited. 


$$
p=\{p(x), x \in S\}
$$

be a distribution on $S$, and

$$
(P(y \mid x)), \quad x, y \in S, \quad t \in T
$$

be stochastic matrices on $S^{2}$. If for each vertex $t \in T$,

$$
\begin{aligned}
& \mathbf{P}\left(X_{t}=y \mid X_{1_{t}}=x \quad \text { and } \quad X_{s} \quad \text { for } \quad t \wedge s \leq 1_{t}\right) \\
& =\mathbf{P}\left(X_{t}=y \mid X_{1_{t}}=x\right)=P(y \mid x), \quad x, y \in S
\end{aligned}
$$

and

$$
\mathbf{P}\left(x_{0}=x\right)=p(x), x \in S,
$$

then $\left\{X_{t}, t \in T\right\}$ will be called $S$-value Markov chains indexed by tree with the initial distribution (1) and transition matrix (2), or called tree-indexed Markov chains.

The above definition is the extension of the definitions of Tree-indexed homogeneous Markov chains [1].

We say a distribution $p>0$ if $p(x)>0$ for all $x \in S$; a stochastic matrix $(P(y \mid x))_{x, y \in S}$ is positive if $P(y \mid x)>0$ for all $x, y \in S$. Denote the distribution of $\left\{X_{t}, t \in T\right\}$ under the probability measure $\mathbf{P}$ by $\mathbf{P}\left(x^{T^{(n)}}\right)=\mathbf{P}\left(X^{T^{(n)}}=x^{T^{(n)}}\right)$. It is easy to see that if $\left\{X_{t}, t \in T\right\}$ is a $S$-valued Markov chains indexed by a tree defined as above, then

$$
\mathbf{P}\left(x^{T^{(n)}}\right)=\mathbf{P}\left(X^{T^{(n)}}=x^{T^{(n)}}\right)=p\left(x_{o}\right) \prod_{t \in T_{(1)}^{n}} P\left(x_{1} \mid x_{1_{t}}\right) .
$$

Definition 2 [2] Let $\left\{X_{\sigma}, \sigma \in T\right\}$ be a collection of $S$-valued random variables defined on $(\Omega, \mathcal{F}), p>0,(P(\gamma \mid x))_{x, y \in S}$ be a positive stochastic matrix, $\mu, \mathbf{P}$ be two probability measure on $(\Omega, \mathcal{F})$, and $\left\{X_{\sigma}, \sigma \in T\right\}$ be Markov chains indexed by tree $T$ under probability measure $\mathbf{P}$. Assume that $\mu\left(x^{T^{(n)}}\right)$ is always strictly positive. Let

$$
\begin{aligned}
& \varphi_{n}(\omega)=\frac{\mu\left(X^{T^{(n)}}\right)}{\mathbf{P}\left(X^{T^{(n)}}\right)} \\
& \varphi(\omega)=\limsup _{n \rightarrow \infty} \frac{1}{\left|T^{(n)}\right|} \ln \varphi_{n}(\omega),
\end{aligned}
$$

then $\phi(\omega)$ will be called the asymptotic logarithmic likelihood ratio.

Remark 1 If $\mu=\mathbf{P}, \phi(\omega) \equiv 0$ holds. Lemma 1 will show that in general case $\phi(\omega) \geq 0$ $\mu$-a.e.; hence, $\phi(\omega)$ can be regarded as a measure of the Markov approximation of the arbitrary random field on $T$.

The tree models have recently drawn increasing interest from specialists in physics, probability, and information theory. Benjamini and Peres [1] have given the notion of the tree-indexed homogeneous Markov chains and studied the recurrence and rayrecurrence for them. Berger and Ye [3] have studied the existence of entropy rate for some stationary random fields on a homogeneous tree. Ye and Berger [4] have studied the asymptotic equipar-tition property (AEP) in the sense of convergence in probability for a PPG-invariant(A PPG is the group of graph automorphisms of $T$ that preserve 
the parities of all vertices. A random field is said to be PPG-invariant if the probability of any finite cylinder set remains invariant under any automorphism of PPG) and ergodic random field on a homogeneous tree. Recently, Yang [5] have studied some strong limit theorems for countable homogeneous Markov chains indexed by a homogeneous tree and the strong law of large numbers and the AEP for finite homogeneous Markov chains indexed by a homogeneous tree. Yang and Ye [6] have studied strong theorems for countable nonhomogeneous Markov chains indexed by a homogeneous tree and the strong law of large numbers and the AEP for finite nonhomogeneous Markov chains indexed by a homogeneous tree. Small deviation theorems are a class of strong limit theorems expressed by inequalities. They are the extensions of strong limit theorems expressed by equalities. It is a new research topic introduced by Professor Liu Wen [7]. Later, Liu and Yang [8] studied the small deviation theorems for the averages of the bivariate functions of $\left\{X_{n}, n \geq 0\right\}$. Liu and Wang [2] studied the small deviation between the arbitrary random fields and the Markov chain fields on Cayley tree. Peng et al [9] established the small deviation theorems for functional of the random fields on homogeneous trees; some of the theorems partly generalized the result of [2].

In this article, the main result can completely generalize the result in [2](Corollary 3). We obtain the small deviation theorems for the random fields and the frequencies of state-ordered couples for random fields on homogeneous trees. Both the result and the method are apparently different from [9]. We arrange the rest of the article as follows:

First, we introduce the asymptotic logarithmic likelihood ratio as a measure of Markov approximation of the arbitrary random field on a homogeneous tree. Second, by constructing a non-negative supermartingale, we obtain a class of small deviation theorems for functionals of random fields on a homogeneous tree. Finally, we establish the small deviation theorems for the frequencies of occurrence of states and ordered couple of states for random fields on a homogeneous tree.

\section{Some Lemmas}

Lemma 1 [2] Let $\mu_{1}, \mu_{2}$ be two probability measures on $(\Omega, \mathcal{F})$, and $D \in \mathcal{F},\left\{\tau_{n}, n \geq 1\right\}$ be a sequence of positive random variables such that

$$
\liminf _{n \rightarrow \infty} \frac{\tau_{n}}{\left|T^{(n)}\right|}>0, \mu_{1}-\text { a.s. on } D \text {. }
$$

Then,

$$
\limsup _{n \rightarrow \infty} \frac{1}{\tau_{n}} \ln \frac{\mu_{2}\left(X^{T^{(n)}}\right)}{\mu_{1}\left(X^{T^{(n)}}\right)} \leq 0, \mu_{1}-\text { a.s. on } D .
$$

Remark 2 Let $\mu_{1}=\mu, \mu_{2}=\mathbf{P}$, by (9) there exists $A \in \mathcal{F}, \mu(A)=1$ such that

$$
\limsup _{n \rightarrow \infty} \frac{1}{\left|T^{(n)}\right|} \ln \frac{\mathbf{P}\left(X^{T^{(n)}}\right)}{\mu\left(X^{T^{(n)}}\right)} \leq 0 \quad \omega \in A ，
$$

and hence we have $\phi(\omega) \geq 0, \omega \in A$. 
Let $k, l \in S, S_{n}(k, \omega)$ (denoted by $S_{n}(k)$ ) be the number of $k$ in the set of random variables $X^{T^{(n)}}=\left\{X_{t}: t \in T^{(n)}\right\}$, and $S_{n}(k, l, \omega)\left(\right.$ denoted by $\left.S_{n}(k, l)\right)$ be the number of couple $(k, l)$ in the set of random couples: $\left\{\left(X_{1_{t}}, X_{t}\right), t \in T\right\}$, i.e.,

$$
\begin{aligned}
& S_{n}(k)=\sum_{t \in T^{(n)}} \delta_{k}\left(X_{t}\right) \\
& S_{n}(k, l)=\sum_{t \in T_{(1)}^{(n)}} \delta_{k}\left(X_{1_{t}}\right) \delta_{l}\left(X_{t}\right),
\end{aligned}
$$

where $\delta_{k}().(k \in S)$ is Kronecker $\delta$-function:

$$
\delta_{k}(x)=\left\{\begin{array}{l}
1, \text { if } x=k \\
0, \text { if } x \neq k
\end{array}\right.
$$

Lemma 2 [2] Let $\mu$ be a probability measure on $(\Omega, \mathcal{F})$, and $\phi(\omega)$ be denoted by (7), $0 \leq c<\ln \left(1-a_{k}\right)^{-1}$ be a constant, and

$$
\begin{aligned}
& D(c)=\{\omega: \varphi(\omega) \leq c\} \\
& M_{k}=\max \left\{\left[\ln \frac{1-a_{k}}{1-\lambda}+c\right] / \ln \frac{\lambda\left(1-a_{k}\right)}{b_{k}(1-\lambda)}, 0<\lambda \leq 1+\left(a_{k}-1\right) e^{c}\right\}
\end{aligned}
$$

where $a_{k}=\max \{P(k \mid i), i \in S\}, b_{k}=\min \{P(k \mid i), i \in S\}$. Then,

$$
\liminf _{n \rightarrow \infty} \frac{S_{n-1}(k)}{\left|T^{(n)}\right|} \geq \frac{M_{k}}{N} \mu-\text { a.e. on } D(c) .
$$

Lemma 3 Let $0<f_{n}(x, y) \leq 1$ be real functions. Then for all $\lambda>0$, we have

$$
\lambda^{f_{n}(x, y)} \leq 1+(\lambda-1) f_{n}(x, y)
$$

Proof It is easy to verify that $\lambda^{f_{n}(x, y)}-(\lambda-1) f_{n}(x, y)-1 \leq 0$ for all $\lambda>0$, the above inequality holds immediately.

Lemma 4 Let $\mu$, $\mathbf{P}$ be two probability measures on $(\Omega, \mathcal{F}), p>0,(P(y \mid x))_{x, y \in S}$ be a positive stochastic matrix, $\left\{X_{\sigma}, \sigma \in T\right\}$ be Markov chains indexed by $T$ under probability measure $\mathbf{P}, 0<f_{n}(x, y) \leq 1$ be real functions defined on $S^{2}, L_{0}=\{o\}(o$ is the root of the tree $T), \mathcal{F}_{n}=\sigma\left(X^{T^{(n)}}\right)$, and $\lambda$ be a real number. Let

$$
\begin{aligned}
& F_{n}(\omega)=\sum_{t \in T_{(1)}^{(n)}} f_{n}\left(X_{1_{t}}, X_{t}\right) \\
& t_{n}(\lambda, \omega)=\frac{\lambda^{F_{n}(\omega)}}{\prod_{t \in T_{(1)}^{(n)}}\left[1+(\lambda-1) E_{P}\left(f_{n}\left(X_{1_{t}}, X_{t}\right) \mid X_{1_{t}}\right)\right]} \frac{\mathbf{P}\left(X^{T^{(n)}}\right)}{\mu\left(X^{T^{(n)}}\right)},
\end{aligned}
$$

where $E_{P}$ is the expectation under probability measure $\mathbf{P}$. Then, $\left(t_{n}(\lambda, \omega), \mathcal{F}_{n}, n \geq 1\right)$ is a non-negative supermartingale under probability measure $\mu$. 
Proof By Lemma 3, we have

$$
\begin{aligned}
& E_{\mu}\left(t_{n}(\lambda, \omega) \mid \mathcal{F}_{n-1}\right) \\
& =t_{n-1}(\lambda, \omega) \sum_{x^{L_{n}}} \frac{\sum_{\lambda^{t \in L_{n}}} f_{n}\left(X_{1_{t}}, x_{t}\right)}{\prod_{t \in L_{n}}\left[1+(\lambda-1) E_{P}\left(f_{n}\left(X_{1_{t}}, X_{t}\right) \mid X_{1_{t}}\right)\right]} \frac{\prod_{t \in L_{n}} P\left(x_{t} \mid X_{1_{t}}\right)}{\mu\left(X^{L_{n}}=x^{L_{n}} \mid X^{T^{(n-1)}}\right)} \times \\
& \mu\left(X^{L_{n}}=x^{L_{n}} \mid X^{T^{(n-1)}}\right) \\
& =t_{n-1}(\lambda, \omega) \sum_{x^{L n}} \frac{\prod_{t \in L_{n}} \lambda^{f_{n}\left(X_{1_{t}}, x_{t}\right)} P\left(x_{t} \mid X_{1_{t}}\right)}{\prod_{t \in L_{n}}\left[1+(\lambda-1) E_{P}\left(f_{n}\left(X_{1_{t}}, X_{t}\right) \mid X_{1_{t}}\right)\right]} \\
& =t_{n-1}(\lambda, \omega) \frac{\prod_{t \in L_{n}} \sum_{x_{t}} \lambda^{f_{n}\left(X_{1_{t}}, x_{t}\right)} P\left(x_{t} \mid X_{1_{t}}\right)}{\prod_{t \in L_{n}}\left[1+(\lambda-1) E_{P}\left(f_{n}\left(X_{1_{t}}, X_{t}\right) \mid X_{1_{t}}\right)\right]} \\
& =t_{n-1}(\lambda, \omega) \prod_{t \in L_{n}} \frac{E_{P}\left(\lambda^{f_{n}\left(X_{1_{t}}, X_{t}\right)} \mid X_{1_{t}}\right)}{\left[1+(\lambda-1) E_{P}\left(f_{n}\left(X_{1_{t}}, X_{t}\right) \mid X_{1_{t}}\right)\right]} \\
& =t_{n-1}(\lambda, \omega) \prod_{t \in L_{n}} \frac{E_{P}\left(\lambda^{f_{n}\left(X_{1^{\prime}}, X_{t}\right)} \mid X_{1_{t}}\right)}{E_{P}\left[1+(\lambda-1) f_{n}\left(X_{1_{t}}, X_{t}\right) \mid X_{1_{t}}\right]} \\
& \leq t_{n-1}(\lambda, \omega) \quad \mu-\text { a.e. }
\end{aligned}
$$

and hence $\left(t_{n}(\lambda, \omega), \mathcal{F}_{n}\right)$ is a non-negative supermartingale under probability measure $\mu$.

\section{Small deviation theorem}

Small deviation theorems are a class of strong limit theorems expressed by inequalities. They are the extensions of strong limit theorems expressed by equalities. It is a new research topic proposed by Professor Liu Wen [9].

In this section, we will establish a class of small deviation theorem for functionals of random fields on homogenous trees. As corollary, we obtain the frequencies of stateordered couples for random fields on homogeneous trees.

Theorem 1 Let $T$ be a homogeneous tree (Bethe tree or Cayley tree), $\mu, \mathbf{P}$ be two probability measures on $(\Omega, \mathcal{F}), p>0,(P(y \mid x))_{x, y \in S}$ be a positive stochastic matrix, $\left\{X_{\sigma}\right.$, $\sigma \in T\}$ be Markov chains indexed by $T$ under probability measure $\mathbf{P}, 0<f_{n}(x, y) \leq \delta_{l}$ $(x) \delta_{k}(y)$ be real functions defined on $S^{2}$, and $\lambda$ be a real number. Let $\phi(\omega)$ and $F_{n}(\omega)$ be denoted by (7) and (15), respectively. Let $c \geq 0$,

$$
\begin{aligned}
& D(c)=\{\omega: \varphi(\omega) \leq c\} \\
& G_{n}(\omega)=\sum_{t \in T_{(1)}^{(n)}} E_{\mathbf{P}}\left(f_{n}\left(X_{1_{t}}, X_{t}\right) \mid X_{1_{t}}\right) .
\end{aligned}
$$

Then,

$$
\limsup _{n \rightarrow \infty} \frac{F_{n}(\omega)-G_{n}(\omega)}{N S_{n-1}(k)} \leq \sqrt{\frac{2 c P(l \mid k)}{M_{k}}}+\frac{c}{M_{k}} \mu-\text { a.e. on } D(c) .
$$


When $0<c<P(l \mid k) M_{k}$,

$$
\liminf _{n \rightarrow \infty} \frac{F_{n}(\omega)-G_{n}(\omega)}{N S_{n-1}(k)} \geq-\sqrt{\frac{2 c P(l \mid k)}{M_{k}}} \quad \mu-\text { a.e. on } D(c) .
$$

Proof Let $t_{n}(\lambda, \omega)$ be defined by (16). By Lemma $4,\left(t_{n}(\lambda, \omega), \mathcal{F}_{n}, n \geq 1\right)$ is a nonnegative supermartingale under probability measure $\mu$. By Doob's martingale convergence theorem, we have

$$
\lim _{n \rightarrow \infty} t_{n}(\lambda, \omega)=t(\lambda, \omega)<\infty \mu-\text { a.e.. }
$$

Hence,

$$
\limsup _{n \rightarrow \infty} \frac{1}{\left|T^{(n)}\right|} \ln t_{n}(\lambda, \omega)=\limsup _{n \rightarrow \infty} \frac{1}{\left|T^{(n)}\right|} \ln t(\lambda, \omega) \leq \mu-\text { a.e.. }
$$

By (14), we get

$$
\limsup _{n \rightarrow \infty} \frac{\ln t_{n}(\lambda, \omega)}{N S_{n-1}(k)} \leq \limsup _{n \rightarrow \infty} \frac{\left|T^{(n)}\right|}{N S_{n-1}(k)} \limsup _{n \rightarrow \infty} \frac{1}{\left|T^{(n)}\right|} \ln t_{n}(\lambda, \omega) \leq 0, \mu-a \text {.e. on } D(c) .
$$

We have by (15), (16) and (21)

$$
\begin{aligned}
& \limsup _{n \rightarrow \infty} \frac{1}{N S_{n-1}(k)}\left[F_{n}(\omega) \ln \lambda-\sum_{t \in T_{(1)}^{(n)}} \ln \left[1+(\lambda-1) E_{\mathbf{P}}\left(f_{n}\left(X_{1_{t}}, X_{t}\right) \mid X_{1_{t}}\right)\right]-\ln \frac{\mu\left(X^{T^{(n)}}\right)}{\mathbf{P}\left(X^{T^{(n)}}\right)}\right] \\
& \leq 0 \mu-a \text {.e. } \quad \text { on } D(c) \text {. }
\end{aligned}
$$

By (6), (7), (17), (22), and $0 \leq c<\ln \left(1-a_{k}\right)^{-1}$, we have

$$
\begin{gathered}
\limsup _{n \rightarrow \infty} \frac{1}{N S_{n-1}(k)}\left[F_{n}(\omega) \ln \lambda-\sum_{t \in T_{(1)}^{(n)}} \ln \left[1+(\lambda-1) E_{\mathbf{P}}\left(f_{n}\left(X_{1_{t}}, X_{t}\right) \mid X_{1_{t}}\right)\right]\right] \\
\leq \frac{c}{M_{k}} \mu-\text { a.e. on } D(c) .
\end{gathered}
$$

By (23) and the inequality $\limsup _{n \rightarrow \infty}\left(a_{n}+b_{n}\right) \leq \limsup _{n \rightarrow \infty} a_{n}+\limsup _{n \rightarrow \infty} b_{n}$, we have

$$
\begin{gathered}
\limsup _{n \rightarrow \infty} \frac{F_{n}(\omega) \ln \lambda}{N S_{n-1}(k)} \leq \limsup _{n \rightarrow \infty} \frac{1}{N S_{n-1}(k)} \sum_{t \in T_{(1)}^{(n)}} \ln \left[1+(\lambda-1) E_{\mathbf{P}}\left(f_{n}\left(X_{1_{t}}, X_{t}\right) \mid X_{1_{t}}\right)\right]+\frac{c}{M_{k}} \\
\mu-\text { a.e. on } D(c) .
\end{gathered}
$$

Let $\lambda>1$, we have by (24),

$$
\begin{gathered}
\limsup _{n \rightarrow \infty} \frac{F_{n}(\omega)-G_{n}(\omega)}{N S_{n-1}(k)} \\
\leq \limsup _{n \rightarrow \infty} \frac{\left.\sum_{t \in T_{(1)}^{(n)}} \ln [1+(\lambda-1)] E_{\mathbf{P}}\left(f_{n}\left(X_{1_{t}}, X_{t}\right) \mid X_{1_{t}}\right)\right]-G_{n}(\omega) \ln \lambda}{N S_{n-1}(k) \ln \lambda} \\
+\frac{c}{M_{k} \ln \lambda} \mu-\text { a.e. on } D(c) .
\end{gathered}
$$


By (17), (25) and inequality $1-1 / x \leq \ln x \leq x-1(x>0)$, we have

$$
\limsup _{n \rightarrow \infty} \frac{F_{n}(\omega)-G_{n}(\omega)}{N S_{n-1}(k)} \leq(\lambda-1) \limsup _{n \rightarrow \infty} \frac{G_{n}(\omega)}{N S_{n-1}(k)}+\frac{\lambda c}{(\lambda-1) M_{k}} \quad \mu-\text { a.e. on } D(c) .
$$

By $0<f_{n}(x, y) \leq \delta_{l}(x) \delta_{k}(y)$, we have $\limsup _{n \rightarrow \infty} G_{n}(\omega) /\left[N S_{n-1}(k)\right] \leq P(l \mid k)$. By (26) we get

$$
\limsup _{n \rightarrow \infty} \frac{H_{n}(\omega)-G_{n}(\omega)}{N S_{n-1}(k)} \leq(\lambda-1) P(l \mid k)+\frac{\lambda c}{(\lambda-1) M_{k}} \quad \mu-\text { a.e. on } D(c) \text {. }
$$

Let $g(\lambda)=(\lambda-1) P(l \mid k)+\lambda c /\left[(\lambda-1) M_{k}\right](\lambda>1)$. In the case $c>0$, (19) holds by (27) because $g(\lambda)$ attains its smallest value $g\left(1+\sqrt{c /\left[P(l \mid k) M_{k}\right]}\right)=2 \sqrt{P(l \mid k) c / M_{k}}+c / M_{k}$ on the interval $(1,+\infty)$; In the case $c=0$, (19) also holds by choosing $\lambda_{i} \rightarrow 1+0(i \rightarrow \infty)$ in (27).

Let $0<\lambda<1$, we have by (24) then

$$
\begin{aligned}
& \limsup _{n \rightarrow \infty} \frac{F_{n}(\omega)-G_{n}(\omega)}{N S_{n-1}(k)} \\
& \geq \liminf _{n \rightarrow \infty} \frac{\sum_{t \in T_{(1)}^{(n)}} \ln \left[1+(\lambda-1) E_{\mathbf{P}}\left(f_{n}\left(X_{1_{t}}, X_{t}\right) \mid X_{1_{t}}\right)\right]-G_{n}(\omega) \ln \lambda}{N S_{n-1}(k) \ln \lambda} \\
&+\frac{c}{M_{k} \ln \lambda} \quad \mu-\text { a.e. on } D(c) .
\end{aligned}
$$

By (17), (28), and inequality $1-1 / x \leq \ln x \leq x-1(x>0)$, we have

$$
\liminf _{n \rightarrow \infty} \frac{F_{n}(\omega)-G_{n}(\omega)}{N S_{n-1}(k)} \geq(\lambda-1) P(l \mid k)+\frac{c}{(\lambda-1) M_{k}} \quad \mu-\text { a.e. on } D(c) \text {. }
$$

Let $h(\lambda)=(\lambda-1) P(l \mid k)+c /\left[(\lambda-1) M_{k}\right](0<\lambda<1)$. In the case $c>0$, (20) holds by (29) because $h(\lambda)$ attains its largest value $h\left(1-\sqrt{c /\left[P(l \mid k) M_{k}\right]}\right)=-2 \sqrt{c P(l \mid k) / M_{k}}$ on the interval (0,1); In the case $c=0$, (20) also holds by choosing $\lambda_{i} \rightarrow 1-0(i \rightarrow \infty)$ in (29). This is the end of the proof.

Corollary 1 Under the assumption of Theorem 1, we have

$$
\lim _{n \rightarrow \infty} \frac{H_{n}(\omega)-G_{n}(\omega)}{N S_{n-1}(k)}=0, \quad \mu-\text { a.e. on } D(0) \text {. }
$$

Proof (30) holds by setting $c=0$ in Theorem 1 .

Corollary 2 Under the assumption of Theorem 1, we have

$$
\lim _{n \rightarrow \infty} \frac{H_{n}(\omega)-G_{n}(\omega)}{N S_{n-1}(k)}=0, \quad P-\text { a.e.. }
$$

Proof Let $\mu=\mathbf{P}$ in Theorem 1, then $\phi_{n}(\omega) \equiv 0, D(0)=\Omega$, and (31) follows immediately.

Corollary 3 (see [2]) Under the assumption of Theorem 1, we have

$$
\limsup _{n \rightarrow \infty}\left[\frac{S_{n}(k, l)}{N S_{n-1}(k)}-P(l \mid k)\right] \leq 2 \sqrt{\frac{c P(l \mid k)}{M_{k}}}+\frac{c}{M_{k}} \quad \mu-\text { a.e. on } D(c),
$$


and when $0 \leq c \leq M_{k} P(l \mid k)$, we have

$$
\liminf _{n \rightarrow \infty}\left[\frac{S_{n}(k, l)}{N S_{n-1}(k)}-P(l \mid k)\right] \geq-2 \sqrt{\frac{c P(l \mid k)}{M_{k}}} \quad \mu-\text { a.e. on } D(c) .
$$

Proof Let $f_{n}(x, y)=\delta_{l}(x) \delta_{k}(y)$ in Theorem 1, then $F_{n}(\omega)=S_{n}(k, l), G_{n}(\omega)=N S_{n-1}(k) P$ $(l \mid k)$, and (32) and (33) hold obviously.

\section{Acknowledgements}

The authors would like to thank the referees for their valuable suggestions and comments. This work is supported by Foundation of Anhui Educational Committee (NO. KJ2012B117), National Natural Science Foundation of China (Grant No. 11071104), the Yang Talents Funds of Anhui (No. 2010SQRL129) and Chaohu University Research Foundation.

\section{Author details}

${ }^{1}$ Department of Mathematics, Chaohu University Chaohu, 238000, P.R. China ${ }^{2}$ Faculty of Science, Jiangsu University Zhenjiang, 212013, P.R. China

\section{Authors' contributions}

WP and WY carried out the design of the study and performed the analysis. PC participated in its design and coordination. All authors read and approved the final manuscript.

\section{Competing interests}

The authors declare that they have no competing interests.

Received: 28 July 2011 Accepted: 28 February 2012 Published: 28 February 2012

\section{References}

1. Benjamini, I, Peres, Y: Markov chains indexed by trees. Ann Prob. 22, 219-243 (1994). doi:10.1214/aop/1176988857

2. Liu, W, Wang, LY: The Markov approximation of the random field on Cayley tree and a class of small deviation theorems. Stat Prob Lett. 63, 113-121 (2003). doi:10.1016/S0167-7152(03)00058-0

3. Berger, T, Ye, Z: Entropic aspects of random fields on trees. IEEE Trans Inform Theory. 36(5):1006-1018 (1990). doi:10.1109/18.57200

4. Ye, Z, Berger, T: Entropic,regularity and asymptotic equipartition property of random fields on trees. J Combin Inform Syst Sci. 21(2):157-184 (1996)

5. Yang, WG: Some limit properties for Markov chains indexed by a homogeneous tree. Stat Prob Lett. 65, 241-250 (2003) doi:10.1016/j.spl.2003.04.001

6. Yang, WG, Ye, Z: The Asympotoic equipartition property for nonhomo-geneous Markov chains indexed by a Homogeneous tree. IEEE Trans Inform Theory. 53(9):3275-3280 (2007)

7. Liu, W: Relative entropy densities and a class of limit theorems of the sequence of m-valued random variables. Ann Prob. 18, 829-879 (1990). doi:10.1214/aop/1176990860

8. Liu, W, Yang, WG: The Markov approximation of the sequences of N-valued random variables and a class of small deviation theorems. Stochast Process Appl. 89, 117-130 (2000). doi:10.1016/S0304-4149(00)00016-8

9. Weicai, Peng, Weiguo, Yang, Wang, Bei: A class of small deviation theorems for functional of random fields on a homogenoous tree. Journal of Mathematical Analysis and Applications. 361, 293-301 (2010). doi:10.1016/j. jmaa.2009.06.079

doi:10.1186/1029-242X-2012-46

Cite this article as: Peng et al:: Markov approximation of arbitrary random field on homogeneous trees. Journal of Inequalities and Applications 2012 2012:46.

\section{Submit your manuscript to a SpringerOpen ${ }^{\circ}$ journal and benefit from:}

- Convenient online submission

- Rigorous peer review

- Immediate publication on acceptance

- Open access: articles freely available online

- High visibility within the field

- Retaining the copyright to your article

Submit your next manuscript at $>$ springeropen.com 